\title{
Growth and light emission of luminous basidiomycetes cultivated on solid media and in submerged culture
}

\author{
Medvedeva $\mathrm{SE}^{1}$, Artemenko $\mathrm{KS}^{2}$, Krivosheenko $\mathrm{AA}^{2}$, Rusinova $\mathrm{AG}^{2}$, Rodicheva \\ EK $^{1}$, Puzyr AP ${ }^{1}$, Bondar VS ${ }^{1,2}$ \\ ${ }^{1}$ Institute of Biophysics SB RAS, Russian Federation, Krasnoyarsk \\ ${ }^{2}$ Siberian Federal University, Russian Federation, Krasnoyarsk
}

Medvedeva SE, Artemenko KS, Krivosheenko AA, Rusinova AG, Rodicheva EK, Puzyr AP, Bondar VS 2014 - Growth and light emission of luminous basidiomycetes cultivated on solid media and in submerged culture. Mycosphere 5(4), 565-577, Doi 10.5943/mycosphere/5/4/9

\begin{abstract}
There are higher fungi that emit visible light; however, little is known about their requirements for good growth and bright luminescence. Knowledge of these requirements is extremely important for maintaining fungal cultures in laboratory conditions and preparation of luminous mycelia for research purposes. Luminous higher fungi Panellus stipticus, Armillaria sp. and Neonothopanus nambi isolated from different climatic areas and maintained in CCIBSO 836 (Collection of IBP SB RAS, Russia) were used for experiments. Techniques for static and submerged cultivation of mycelia of higher fungi have been developed and optimized for the production of samples of aerial and globular mycelia with prolonged and stable luminescence. We investigated the growth characteristics and luminescence of mycelia cultivated in/on different nutrient media, and the effects of deionized water and mechanical damage on the light emission of mycelia. An increase in luminescence intensity of fungal mycelia can be obtained during cultivation of fungi on a nutrient medium with a certain composition. A significant increase in light emission from $N$. nambi mycelium can also be obtained after its incubation in water and mechanical damage. The light emission from N. nambi mycelium was greatly enhanced after these treatments, in contrast to the mycelia of Armillaria sp. or P. stipticus. Cultivation conditions that enable growing mycelia with high levels of luminescence will expedite further studies to gain a better understanding of fungal bioluminescence.
\end{abstract}

Keywords - luminescence - luminous higher fungi - mycelium

\section{Introduction}

There are more than 80 species of luminous higher fungi that emit visible light (Shimomura 2006, Desjardin et al. 2008, 2010, Vydryakova et al. 2009). Luminous fungi are generally saprobes (less frequently, pathogens) of plants. The glowing fungi grow on rotting wood and their light is visible at night; this effect prompted the popular names "shining wood" and "fox fire" (Harvey 1952, 1957). Luminous higher fungi emit greenish light with a maximum emission in the range of 520-530 nm (O'Kane et al. 1990, Shimomura 2006, Desjardin et al. 2008, Bondar et al. 2011). A luminous taxon emits light in only a certain period of its life cycle; before and after that period, it generally does not glow. For instance, in representatives of the genera Mycena and Omphalotus, 
both the mycelium and fruiting body can glow, whereas in species of Armillaria, only the mycelium and rhizomorphs glow (Shimomura 2006). In general, the luminescence of younger fruiting bodies and young actively growing mycelia is brighter than that of mature fruiting bodies and old mycelia, even though the intensity of their luminescence varies widely with the species and the environment (Shimomura 2006, Desjardin et al. 2008, Bondar et al. 2012). Despite the fact that the intensity of their luminescence is generally low compared with that of many other luminescent organisms, fungi glow continuously for several days.

To reliably maintain luminous fungal cultures, researchers should choose the most appropriate nutrient medium on which the mycelium has good growth and high levels of luminescence during prolonged storage. Therefore, the development of methods for cultivating luminous fungi under laboratory conditions is one of the main goals among investigations of the bioluminescent fungi (Endo et al. 1970, Bermudes et al. 1990, Niitsu et al. 2000, Weitz et al. 2001, Mendes et al. 2008, Dao 2009, Prasher et al. 2012, Bondar et al. 2013). Knowledge of these requirements is extremely important not only for maintaining fungal cultures under laboratory conditions but also for preparation of luminous mycelia for various research purposes.

The aims of our study were to choose the optimal nutrient medium for growing luminous mycelia of different fungal species under laboratory conditions and find a way to enhance luminescence. We studied the relationships between the growth and luminescence of mycelia grown on nutrient media with different compositions and the effect of de-ionized water and mechanical damage on the light emission of the mycelia.

\section{Materials \& Methods}

Three species of luminous higher fungi isolated from different regions were used in this study. Their cultures are available in the CCIBSO 836 collection of the Institute of Biophysics, Siberian Branch of Russian Academy of Sciences. They are

1. Neonothopanus nambi (Speg.) R.H. Petersen \& Krisai (IBSO 2307) - isolated in 2000 by the Vietnamese researcher Dao Thi Van in the subtropical forests of Southern Vietnam.

2. Armillaria sp. (IBSO 2310) - isolated in 2007 by N.V. Pashenova (Institute of Forest, Siberian Branch of Russian Academy of Sciences) in the suburbs of Krasnoyarsk (Russia) in an area with a clear continental climate. The specific feature of the strain studied was a very low ability to produce rhizomorphs during growth on any medium.

3. Panellus stipticus (Bull.: Fr.) P. Karst (IBSO 2301) - isolated in 2010 by G.A. Vydryakova in the suburbs of Ottawa (Canada) in an area with a continental climate.

The cultures were maintained on malt-extract agar slants; Armillaria sp. and Panellus stipticus were kept refrigerated at $+4^{\circ} \mathrm{C}$ and Neonothopanus nambi at $+18^{\circ} \mathrm{C}$. Techniques for the static and submerged cultivation of these selected luminous higher fungi were developed and optimized for the production of samples of aerial and globular mycelia with a prolonged and stable level of luminescence. The aerial mycelial biomasses were grown in Petri dishes on liquid and solid nutrient media with different compositions.

The following media were used for the study of mycelial growth and luminescence: Maltextract medium (ME) (glucose $2 \mathrm{~g} / \mathrm{L}$, malt-extract $20 \mathrm{~g} / \mathrm{L}$, peptone $1 \mathrm{~g} / \mathrm{L}$ ); Potato-glucose medium (PG) (potato broth $200 \mathrm{~g} / \mathrm{L}$, glucose $20 \mathrm{~g} / \mathrm{L}$ ); Yeast-extract - Malt-extract medium (YM) (yeastextract $3 \mathrm{~g} / \mathrm{L}$, malt-extract $3 \mathrm{~g} / \mathrm{L}$, glucose $10 \mathrm{~g} / \mathrm{L}$, peptone $5 \mathrm{~g} / \mathrm{L}$ ); Sabouraud medium (Sab) (mycological peptone $10 \mathrm{~g} / \mathrm{L}$, glucose $40 \mathrm{~g} / \mathrm{L}$ ); Fungal medium (F) (papain digest of soya flour 10 $\mathrm{g} / \mathrm{L}$, glucose $10 \mathrm{~g} / \mathrm{L}$ ). The above-mentioned nutrient media were produced by HiMediaLabs, India. Potato-sucrose medium (PS) (potato broth $200 \mathrm{~g} / \mathrm{L}$, sucrose $20 \mathrm{~g} / \mathrm{L}$ ) was also used. The solid nutrient media contained also 15-20 g/L agar (Difco or HiMedia). All the media were autoclaved at $121^{\circ} \mathrm{C}$ for $15 \mathrm{~min}$ immediately prior to use.

The mycelia of Panellus stipticus and Armillaria sp. were grown at $22-23^{\circ} \mathrm{C}$, and the mycelia of Neonothopanus nambi were grown at $27^{\circ} \mathrm{C}$. The growth of the mycelia was evaluated by the 
standard method of measuring the diameter increase of colonies under cultivation on an agar substrate in $35 \mathrm{~mm}$ diameter Petri dishes.

The luminous fungus Neonothopanus nambi was the main material for submerged cultivation. The mycelia were grown in 100-500 ml conical vessels containing liquid nutrient PS medium (30-200 ml). The mycelium was preliminarily grown for 8-10 days on Petri dishes (90 $\mathrm{mm}$ diameter) in a liquid nutrient medium and used as the inoculum for submerged culture. The volume of the seeding material was $2-5 \%$ of the volume of the nutrient medium. Cultivation was conducted for 3-4 days at $27-28^{\circ} \mathrm{C}$ with continuous stirring at $180-200 \mathrm{rpm}$. The mycelia of other fungi were cultivated under similar conditions in the optimal medium for each fungus. The mycelia of Panellus stipticus and Armillaria sp. were grown at $22-23^{\circ} \mathrm{C}$.

In all the experiments, the intensity of the light emission of the mycelial samples was determined as relative light units per second (RLU/s) using a GloMax 20/20 luminometer (Promega, USA). All the growth and luminescence measurements were performed at the same time (between $12.00 \mathrm{am}$ and $2 \mathrm{pm}$ ). Three replicates were used for each medium in three independent experiments. Images and luminescence of the mycelial samples were also recorded using a Universal Hood II system (Bio-Rad, USA), which makes it possible to obtain an image of the sample in white light and record its luminescence in the signal accumulation mode in the dark.

Statistical analysis was carried out with statistical analysis package MS Office Excel. The significance levels expressed were at a $95 \%$ confidence level.

\section{Results}

\section{Growth of fungal mycelia during cultivation on solid media with different compositions}

The mycelia of the luminous fungi exhibited different growth activity under cultivation on solid media. The Neonothopanus nambi mycelium generally exhibited higher growth activity on solid medium than the mycelia of Panellus stipticus and Armillaria sp. (Fig. 1). The initial disc of Neonothopanus nambi mycelium increased 2 to 5-fold as early as day 3 after inoculation on almost all media. The mycelial colonies in most cases reached a maximum size (complete coverage of the entire surface of the agar medium in the $35 \mathrm{~mm}$ Petri dish) on days 7 and 10 of cultivation (Fig. 1). Nevertheless, the medium composition was important for growth of Neonothopanus nambi colonies. It was shown that the mycelium grew faster on PSA than on the other media used. Colonies grown on PSA were denser, and therefore, higher biomass was produced.

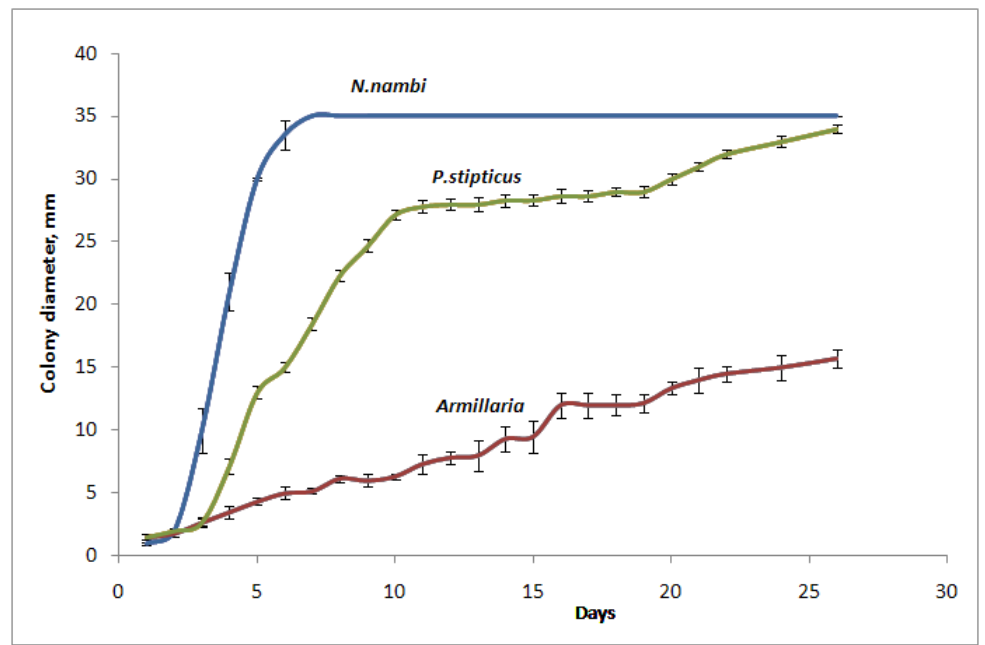

Fig. 1 - Growth dynamics of fungal mycelia on the PGA. The mycelia growth for each fungus had these typical properties with some differences depending on the composition of the medium. Neonothopanus nambi and Panellus stipticus mycelia colonized the entire substrate uniformly and had slight differences in colony thickness on the media studied; for Armillaria sp., the height and size of colony were more substantial. 
Cultivation of Panellus stipticus mycelia on solid media did not reveal any considerable differences between the growth rates of colonies on media with different compositions. Complete coverage of the entire surface of the agar medium in the $35 \mathrm{~mm}$ Petri dish was observed usually on the $23^{\text {rd }}$ to $25^{\text {th }}$ days of cultivation (Fig. 1). The growth of the mycelia on all the solid media was accompanied by the formation of dense and planar colonies, except on PSA, on which the colonies were less dense and had very fine hyphae.

Under static cultivation conditions, the Armillaria sp. mycelial colonies exhibited nearly linear growth on solid media (Fig. 1). The mycelia of this fungus showed the best growth on PGA, MEA and SabA; at the same time, the mycelial colonies on SabA were denser than those on PGA. It should be noted that the Armillaria sp. mycelia in colonies grown on PSA had numerous peripheral hyphae that grew diffusely on the substrate surface. As a result, the edges of the colonies had no clear boundaries. On YMA and FA, we observed the growth of dense high mycelial colonies with clear-cut edges. Complete coverage of the entire surface of the agar medium in the 35 mm Petri dish was not observed during 25 days of cultivation (Fig. 1).

Thus, it was shown that the Neonothopanus nambi mycelium exhibited higher growth activity on all media than the mycelia of Panellus stipticus and Armillaria sp.; and the mycelia of Armillaria sp. grew substantially slower in comparison with other fungal mycelia studied.

\section{Luminescence of fungal mycelia during cultivation on solid media with different compositions}

Luminescence of mycelial colonies varied between representatives of different fungal species and depended on the medium composition. Panellus stipticus mycelia had a higher luminescence level compared with luminescence of other mycelia studied on almost all media. The greatest light emission was obtained when Panellus stipticus mycelia were grown on PGA and SabA (Fig. 2). The difference in the level of light emission of fungal mycelia can be seen during observation of glowing colonies in the darkness with the unaided eye or with a special device (Fig. 3).

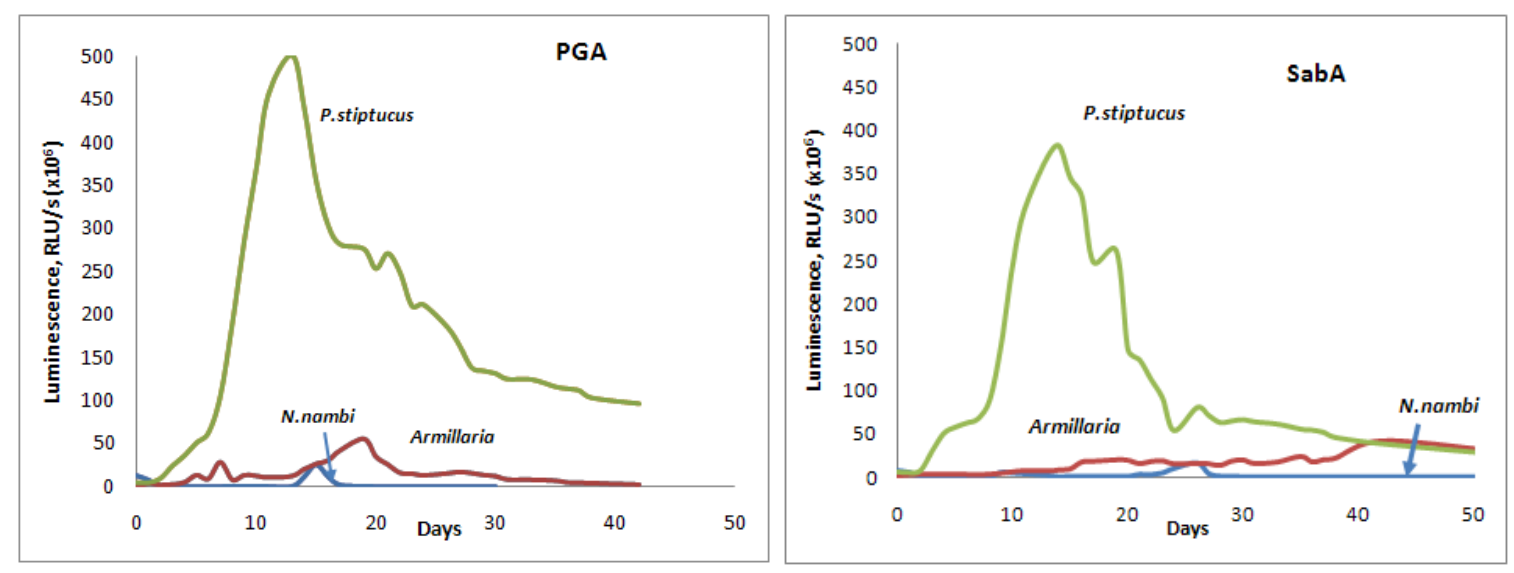

Fig. 2 - Variations in luminescence of the fungal mycelia grown on PGA and SabA. The cultivation period was more than 40 days for each culture at optimal temperature.

Comparison of luminescence levels in the fungal mycelia grown on media with different compositions is shown in Fig. 4. The highest levels of light emission were observed for Panellus stipticus mycelia; Neonothopanus nambi mycelia had low luminescence intensity on practically all media, excluding PSA. Armillaria sp. mycelia showed an intermediate position. From the data in Fig. 4, it can be seen that luminescence levels were low under cultivation of all fungal mycelia studied on the FA. Armillaria sp. and Panellus stipticus mycelia revealed several luminescence peaks under cultivation on all media while a single peak of light emission was more characteristic for Neonothopanus nambi mycelia. The mycelia of each fungus had its own maximum of light emission intensity (Fig. 4). From these data, we can see that mycelia of the fungi studied reached 
the maximum light emission levels at different times. We observed the coincidence of the peaks in luminescence only in some cases for Panellus stipticus and Neonothopanus nambi grown on YMA (Fig. 4).

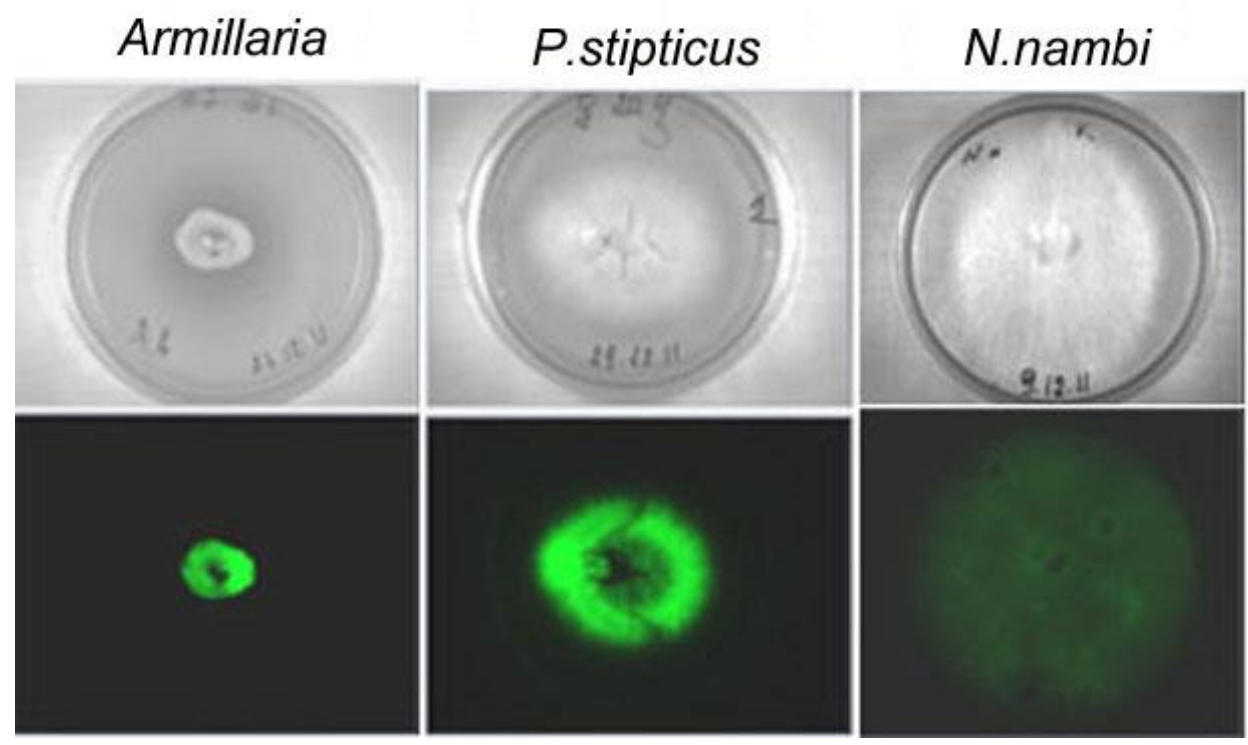

Figs. 3 - Fungal mycelia grown in Petri dishes ( $35 \mathrm{~mm}$ diameter) on PGA during 10 days. Colonies in daylight (top), and their light emission in the dark (bottom) recorded by using Universal Hood II system (Bio-Rad, USA). Exposure - $300 \mathrm{~s}$.

The luminescence intensity of the mycelial colonies changed during their growth on solid media. The most prolonged light emission by the Neonothopanus nambi mycelium at a high intensity (near 20 days) was observed when it was cultivated on PSA. The luminescence that can be seen with the unaided eye on this medium was recorded at 8-10 days and lasted up to 35-37 days (Fig. 4, PSA). When the mycelium was grown on PGA, SabA, YMA or FA, bright luminescence of mycelial colonies was recorded within a certain number of days and only for short (up to 3-4 days) periods. When the Neonothopanus nambi mycelium was grown on MEA, its luminescence reached a maximum on the $12^{\text {th }}$ day of cultivation, decreasing afterward, and persisting for a long period at an extremely low intensity (Fig. 4, MEA). Our observations indicated that visible glow was observed in darkness in Neonothopanus nambi mycelia when the growth of the colony slowed down or stopped (Fig. 5). Biomass accumulation occurs with different growth rates on media with different compositions, which explains the occurrence of luminescence at different times of cultivation and with varying intensity. Panellus stipticus mycelium growing on all of the media exhibited long-term luminescence (more than 40 days) (Fig. 2, 4). Although the luminescence differed in the time of occurrence and intensity, its maximum was reached at certain times depending on the composition of the culture medium: after 4-5 days of cultivation on FA, after 911 days of cultivation on PSA, after 11-14 days on PGA, after 10-11 days of cultivation on MEA, after 13-15 days on SabA and YMA. As noted above, Panellus stipticus mycelia did not reveal any considerable differences in growth of colonies on media with different compositions.

Cultivation of Armillaria sp. mycelia for long periods (up to 50 days and longer) showed that the colonies exhibited prolonged luminescence and that the intensity considerably depended on the growth time and the composition of the medium (Fig. 4). It was apparent that the luminescence of the Armillaria sp. colonies reached a maximum luminescence level as early as days 5-6, when the mycelia were grown on YMA, after 12-15 days of cultivation on PSA and MEA, near 20 days on PGA and after 40 days on SabA. Armillaria sp. mycelia grown on PSA and FA media had the weakest luminescence compared to that of the mycelia grown on other media. 

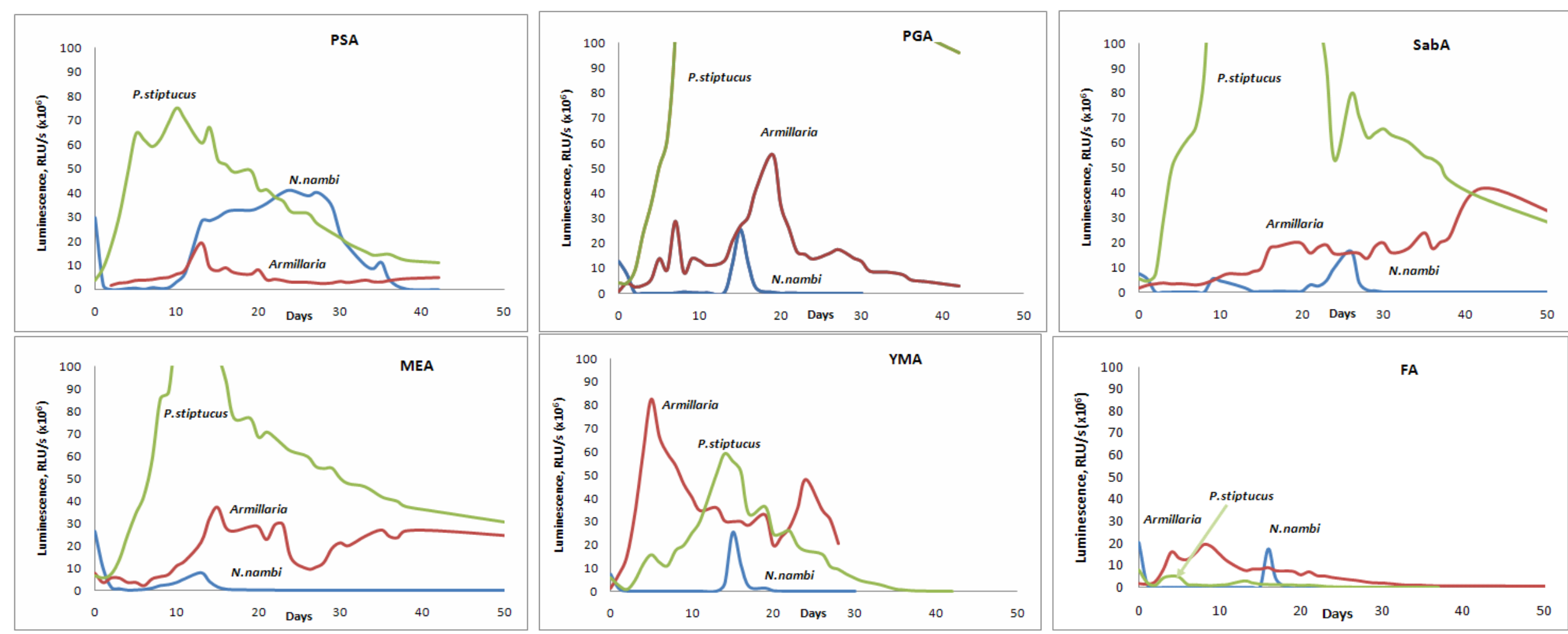

Figs. 4 - Typical dynamics of luminescence intensity of fungal mycelia grown on solid media with different compositions: PSA - potato-sucrose agar, PGA - potato-glucose agar, SabA - Sabouraud dextrose agar, MEA - malt agar, YMA - malt and yeast extract agar, FA - fungal agar. The cultivation period was more than 40 days for each culture at optimal temperature. 


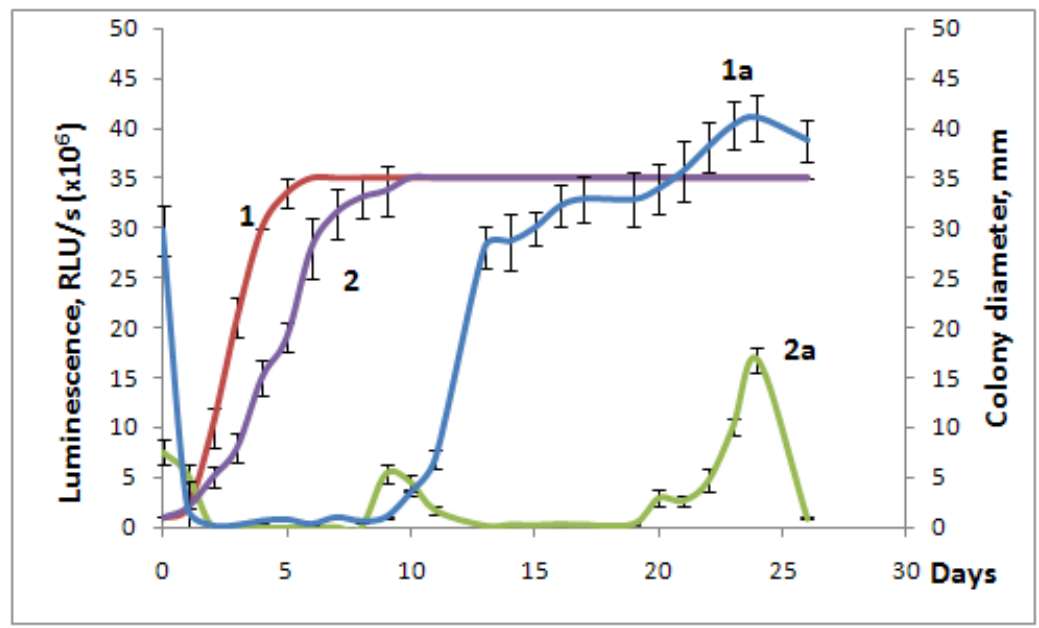

Fig. 5 - Luminescence of Neonothopanus nambi mycelium became observable in darkness after the mycelial growth slowed down or stopped. Growth $(1,2)$ and luminescence intensity $(1 \mathrm{a}, 2 \mathrm{a})$ of mycelia grown on PSA (1, 1a) and SabA $(2,2 a)$ media.

\section{Submerged culture of luminous fungi}

Submerged Neonothopanus nambi culture was grown in PS broth for $3-4$ days at $27-28^{\circ} \mathrm{C}$ with continuous stirring at $180-200 \mathrm{rpm}$. The mycelia of other fungi were cultivated under similar conditions in the suitable (see above) media, at the optimal temperature and optimal time for each fungus. The mycelia of Panellus stipticus and Armillaria sp. were grown at $22-23^{\circ} \mathrm{C}$. The process that was developed allowed the production of mycelial biomasses of fungi in the form of spherical pellets (globules). Mycelial pellets of Neonothopanus nambi in submerged culture had a very rough surface due to the numerous outgrowths. Using this cultivation technique, the diameter of the mycelial globules that were produced ranged from 2 to $7 \mathrm{~mm}$ (Fig. 6A).

The globules of Neonothopanus nambi mycelia submerged in liquid nutrient medium exhibited low-level luminescence, only 3-10 times higher than the background noise of the measurement system (50-62 RLU/s). However, incubating Neonothopanus nambi globules in deionized water for 14-18 hours substantially increased their luminescence (an increase of up to 1000 times) for a long period of time (several days). The luminescence also increased when the pellets that were submerged in water were bubbled with air (Fig. 6 B-D).
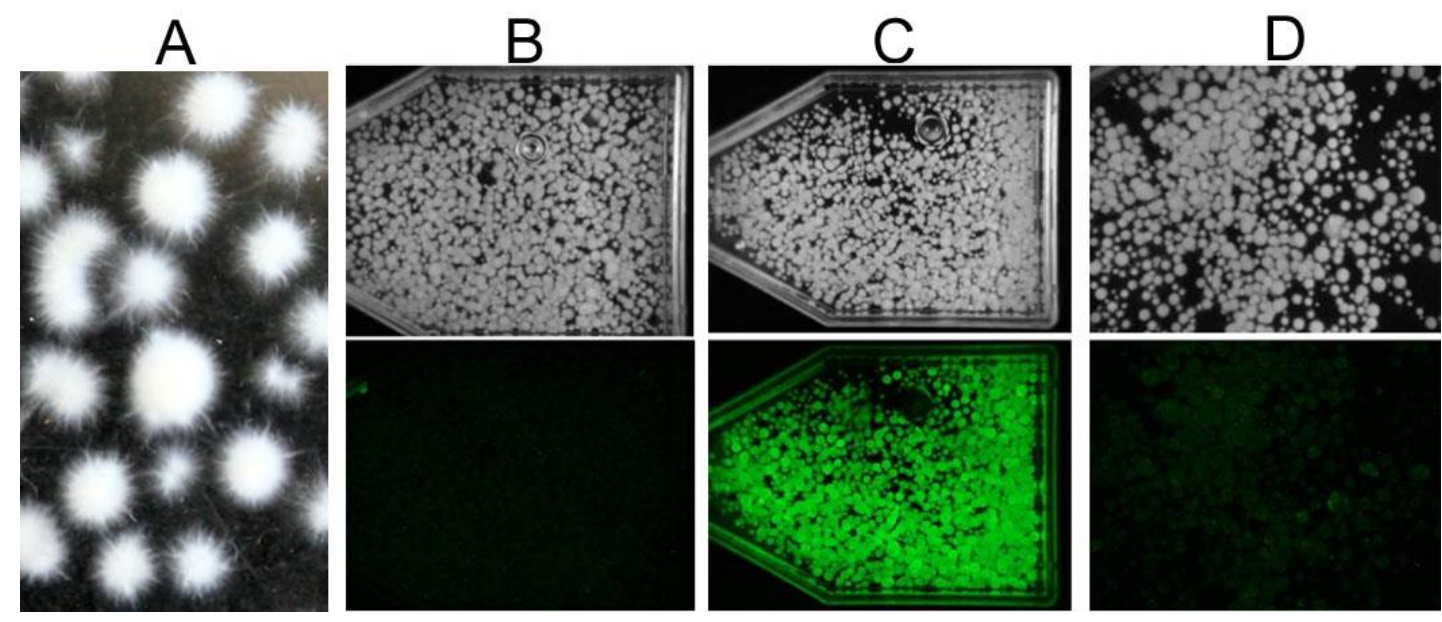

Figs. 6 - Mycelial pellets of Neonothopanus nambi in submerged culture with very rough surface A

- External appearance (top) and luminescence (bottom) of the pellets in processing the $N$. nambi 
mycelia pellets with deionized water: $\mathrm{B}$ - before washing with deionized water, $\mathrm{C}-$ maximum luminescence after washing with deionized water, D - low luminescence after prolonged washing with deionized water. Images made by Universal Hood II (USA). Exposure: B, D - 600 s, C - 100 s.

Our studies showed that the pellets of Neonothopanus nambi demonstrated strikingly bright luminescence (increasing more than 1000 times) only after exposure to deionized water, but the Armillaria sp. pellets that were submerged in liquid nutrient medium already had bright and longlasting luminescence (Fig. 7). Their luminescence increased inconsiderably after incubation in deionized water - approximately 2 to 5-fold only.
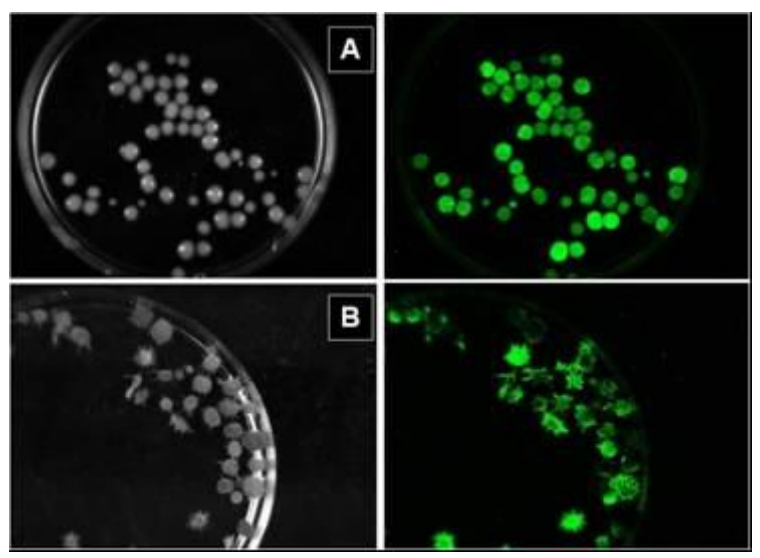

Figs. 7 - Pellets of fungi (left) and their light emission (right) in submerged conditions: A Neonothopanus nambi globules after incubation in deionized water for 16 hours; B - Armillaria sp. globules in nutrient medium. Images made by Universal Hood II (USA). Exposure - $300 \mathrm{~s}$.

The same effect was observed when aerial mycelial discs were incubated in deionized water. Mycelial pellets of Panellus stipticus in submerged culture were globules with rough surface similar to Neonothopanus nambi pellets, but their light emission was not registered by a special device even after incubation in deionized water.

\section{The luminescence response to mechanical stress}

We also studied the luminescence response of fungal mycelia to mechanical stress. It was found that mechanically damaged mycelia caused a sharp and rapid increase in the luminescence level of Neonothopanus nambi but a weak response in light emission of Armillaria sp. (Fig. 8). Mechanical damage of Neonothopanus nambi mycelia (incision of the colony) initiated an increase in light emission that developed within 1-3 hours and lasted for 3-5 days. The luminescence response to mechanical damage was much less significant for Armillaria sp. and Panellus stipticus mycelia.
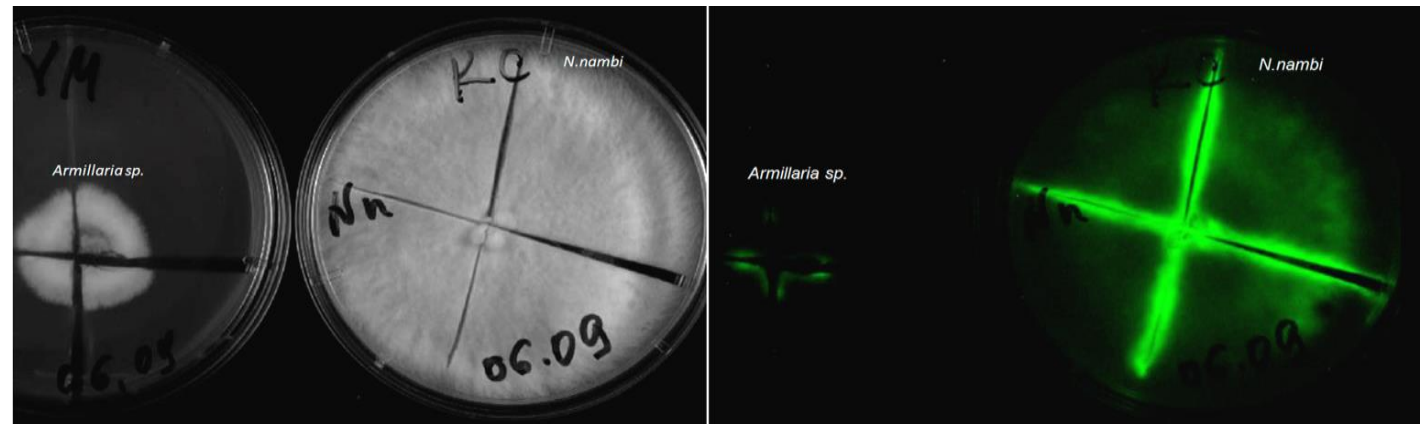

Figs. 8 - Mechanical damage increased the luminescence of Neonothopanus nambi. Fungal colonies (left) and their luminescence after incision of the colony (right). Images made by Universal Hood II (USA).Exposure - $300 \mathrm{~s}$. 
The luminescence of Armillaria sp. mycelium increased by 7-10 times at 1.5-2.5 hours after mechanical damage and then decreased to the baseline level. Panellus stipticus mycelium reacted to damage in another way; initially the luminescence decreased 2 to 3 -fold, then increased to a certain value and remained at this level for a long time. The strongest response to injury was observed in the Neonothopanus nambi mycelia. Already 1 hour after incision of the colony, the luminescence had increased 5-fold, reaching a maximum after 3-4 hours (a 20-fold increase) when the colonies were growing on agar (Fig. 9).

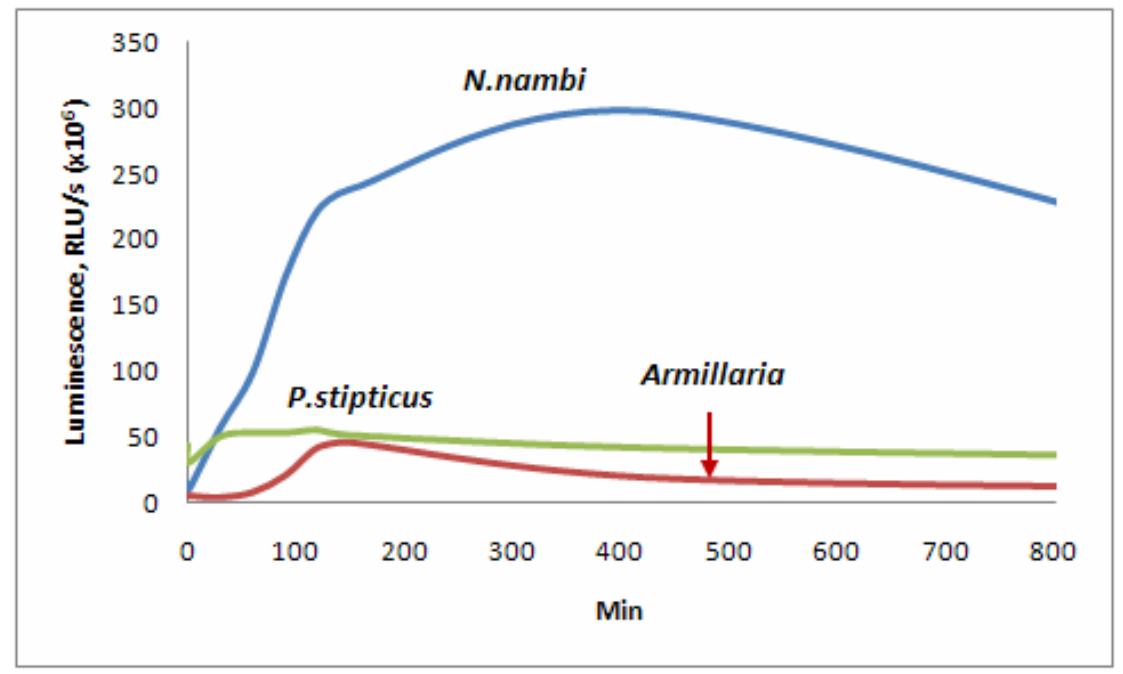

Fig. 9 - The mechanical damage of mycelia caused different responses in luminescence of Neonothopanus nambi, Armillaria sp. and Panellus stipticus mycelia. The colonies were incised with a scalpel.

Mechanical damage of mycelia can be used for "kindling" pellets of Neonothopanus nambi that have lost their luminescence (Fig. 10). If the pellets that had lost their luminescence were cut into several pieces, their luminescence appeared again after some time.

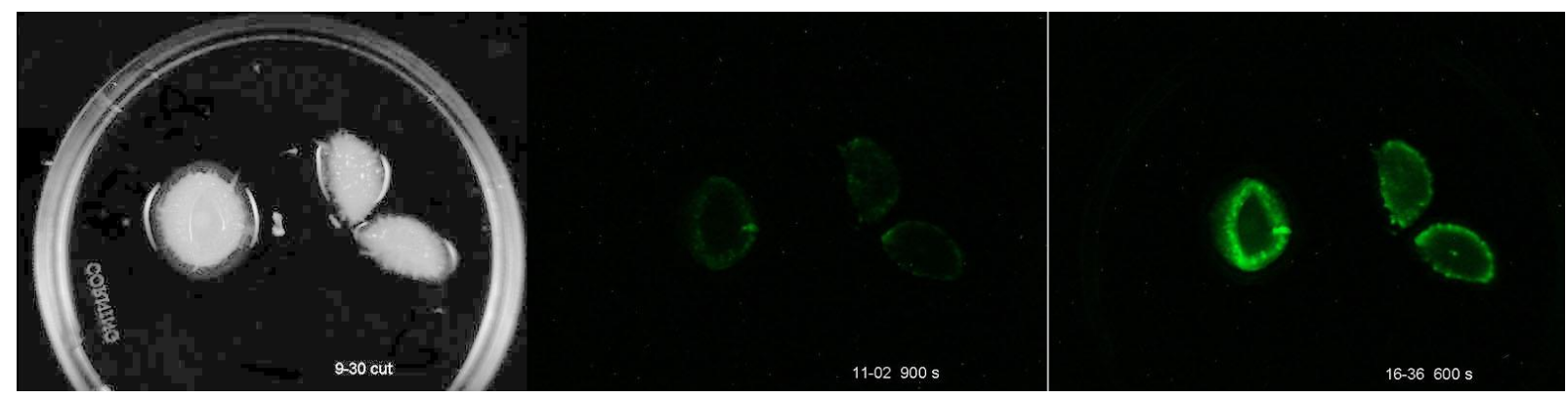

Figs. 10 - Non-luminous pellets were cut into several pieces (left), and the glow re-appeared (middle), reaching a maximal level after 7 hours (right). Images made by Universal Hood II (USA). Exposure: middle $-900 \mathrm{~s}$, right $-600 \mathrm{~s}$.

\section{Discussion}

In preliminary experiments, we tried to use synthetic nutrient medium to obtain sufficient mycelium biomass with high luminescence. However, the nutrient medium without complex organic compounds (peptone, yeast extract or malt) did not give the desired result. Therefore, we used agar-based media with different compositions of organic components, which are widely used 
for the isolation, cultivation and enumeration of yeasts and molds present in different products and beverages (Bekker 1988). Mycelial cultures are routinely maintained on agar-based media, such as malt extract agar and yeast-malt extract agar containing peptone and dextrose in fungal collections (Psurtseva et al. 2007).

Examination of relationships between the growth and luminescence of the luminous organisms is one of the main issues in studying the luminescence mechanism. There are several reports describing the coordinated increase in mycelial growth and luminescence for certain types of fungi (Bermudes et al. 1990, Weitz et al. 2001, Shimomura 2006, Mendes et al. 2008, Dao 2009). In our experiment, Neonothopanus nambi mycelia had similar characteristics to those reported by Dao (2009). Dao studied samples that were cut from Neonothopanus nambi mycelia. She obtained the rapid growth of Neonothopanus nambi colonies on YMA and MEA and bright luminescence on Hamada medium. We measured the luminescence of intact mycelia; the Neonothopanus nambi colonies increased faster and glowed brightly under cultivation on PSA (Figs 4, 5).

Previous studies of growth and total light emission in Panellus stipticus mycelium grown on solid media showed that luminescence occurred after 4 days of cultivation, while after 13 days of cultivation it decreased and then increased after 18 days. Optimum luminescence level was attained after 24 days of cultivation (Prasher et al. 2012). In contrast, our studies demonstrated that luminescence of Panellus stipticus mycelium could be seen with the unaided eye in darkness after 3-6 days of cultivation on all the solid media studied. Its light emission increased to maximal level without any decrease. Panellus stipticus colonies were grown on media with various compositions in dark conditions, and there were only small differences in their growth characteristics. Panellus stipticus showed rapid mycelial growth and bright luminescence on PGA, SabA and MEA, but the luminescence intensity of mycelia was slightly lower during cultivation on MEA (Fig. 2, 4).

Mihail \& Bruhn (2007) observed the lowest luminescence intensity for Armillaria tabescens, the species with the highest linear growth rate. They remarked that the luminescence of Armillaria species was responsive to the medium composition, although the luminescence of Armillaria tabescens mycelia fluctuated more than that of Armillaria mellea. Weitz with co-authors (2001) observed the linear growth for Armillaria mellea mycelia grown on MEA. We also found that colonies of Armillaria sp. mycelia increased linearly on all solid nutrient media. Armillaria sp. had rapid mycelial growth on PGA, SabA and MEA, whereas their bright luminescence was observed on YMA and PGA. Maximum of light emission level occurred at different times, and there were often a few peaks of luminescence. The luminescence of Armillaria sp. colonies of the same age was bright in the small colonies on YMA medium and the rapidly growing colonies on PGA, SabA and MEA. However, the luminescence reached its maximum levels at different times, which are specific for each medium (Fig. 4). In our experiments, the strain of Armillaria sp. studied showed maximal luminescence earlier than Armillaria mellea (Weitz et al. 2001), when mycelia were grown on MEA medium. We also found that luminescence reached a maximum value earlier on medium containing yeast and malt extracts than on other media, but the colonies grown on YMA medium were smaller than those grown on other media.

Thus, Armillaria sp. formed compact and dense colonies with a high-intensity prolonged luminescence level. Panellus stipticus formed colonies with a high-intensity prolonged luminescence level, but the colonies were larger than those of Armillaria sp. Neonothopanus nambi mycelia grew more rapidly than the mycelia of Armillaria sp. and Panellus stipticus, but the luminescence of Neonothopanus nambi was substantially lower than the light emission of Armillaria sp. and Panellus stipticus mycelia. The mycelial growth and luminescence characteristics of Neonothopanus nambi and Panellus stipticus that were observed in this study were similar to those reported in other studies, but these characteristics for Armillaria sp. had some differences from those known for Armillaria mellea, Armillaria. gallica and Armillaria tabescens. Therefore, the growth and luminescence level of mycelia of fungi from different habitats and different taxa are significantly different. It may be assumed that each fungus generates certain 
requirements for a medium in its natural habitat, and it requires these conditions under cultivation in vitro.

The important elements for fungal metabolism are the carbon sources, which the fungus can use via an accessible form in the substrate or which the fungus can produce from the components in the substrate through enzymatic activity. The commonly used carbon sources include monosaccharides, potato dextrose, Yeast extract, and malt extract (Bekker 1988). The enzyme systems capable of utilizing the organic compounds present in the substrates have been found in fungi (Mane et al. 2007). It has been shown that media lacking added carbon sources supported extremely sparse radial growth which gave very low levels of luminescence and, thus, are less suitable to be used in growing of Panellus stypticus cultures (Bermudes 1990). In our experiments, Neonothopanus nambi mycelia grew well and had high and sustained levels of luminescence on media containing organic compounds and glucose in the bound state (the medium containing potato broth and sucrose). On media containing glucose in the initially accessible form, the luminescence did not last as long and did not reach the levels observed on PSA. The mycelia on the medium with glucose content of 2-5 g/L began to glow after 7-10 days. At higher glucose concentrations glow of mycelium started only after 2-3 weeks. The mycelia of the other fungi that were studied exhibited certain differences from Neonothopanus nambi mycelium. Panellus stipticus showed rapid mycelial growth on PGA, SabA and MA, with the brightest luminescence displayed on PGA and SabA. The luminescence was less intense during cultivation on MEA. Armillaria sp. showed rapid mycelial growth on PGA, SabA and MEA, whereas bright luminescence was observed on YMA and PGA. Maximum luminescence occurred at different times, and there were often a few peaks of luminescence (Fig. 4).

There is information that submerged culture of luminous fungal mycelia can be used in bioassays (Weitz et al. 2002). Mycelium of some fungi grown in submerged culture conditions may glow, but not all fungi have this property. For example, pellets of Mycena and Armillaria mellea were glowing (Kamzolkina et al. 1983, Weitz et al. 2002). It was found that Panellus stipticus was non-luminous when it was submerged in defined liquid media with or without agitation (Bermudes et al. 1990, Shimomura 2006). However, Prasher and co-authors (2012) found that Panellus stipticus had a very low intensity luminescence on the $13^{\text {th }}$ day of growth in liquid media. We did not detect any luminescence of globular Neonothopanus nambi mycelium taken from a culture medium, i.e., the level of luminescence was not significantly different from the background noise of the measuring system (Bondar et al. 2013). Incubation of Neonothopanus nambi mycelial globules in deionized water for 12-24 h resulted in a significant increase in the level of luminescence (Fig. 6). The globular mycelium has several advantages as material for scientific studies. The technique of submerged cultivation allows producing globular mycelia with a diameter of 2-7 mm and more, according to experimental tasks. Researchers can choose globules of the uniform size suitable for their purposes. The globules can be easily transferred using a spatula from one liquid medium into another as well as to the measuring cell in a luminometer or another device. Fungal mycelia undergo minimal mechanical stress and damage in this process.

The response of some Armillaria species to mechanical disturbance events was studied by Mihail \& Bruhn (2007). They found that luminescence of Armillaria gallica mycelium was greatly enhanced by a single disturbance event; the effect lasted at least $4 \mathrm{~h}$. The response of Armillaria mellea and Armillaria tabescens mycelial luminescence to mechanical disturbance was much less consistent than that observed for Armillaria gallica. When we examined the luminescence response to mechanical damage of mycelia, we observed that the luminescence of Neonothopanus nambi mycelia was greatly enhanced compared with that of Armillaria sp. and Panellus stipticus mycelia. Mechanical damage of Neonothopanus nambi mycelium (incision of the colony) initiated the increase in light emission that developed within 1-3 hours and lasted for 3-5 days. The effect was much less significant for luminescence in Armillaria sp. and Panellus stipticus mycelia (Fig. 9).

The fungi studied were isolated from different substrates in different parts of the world from subtropical areas to areas with a continental or extreme continental climate. The study showed that the mycelia of each fungus required individual nutrient media to grow and produce light 
emission of high intensity. Therefore, the growth and luminescence level of fungal mycelia from different habitats and different taxa are significantly different. Several methods of enhancing the luminescence of mycelia were found: a suitable composition of the nutrient medium, incubation in water and mechanical damage. Characteristics of Neonothopanus nambi and Armillaria sp. pellets observed in this study have not been described in previous works. The nutrient media tested will facilitate maintaining the fungal cultures in the CCIBSO collection. They will also be used to expedite further studies for better understanding of fungal bioluminescence.

\section{Acknowledgments}

This study was supported by grant No. 11.G34.31.058 (RF Government) and Projects No. 71 and No. 38 (SB RAS).

\section{Conflicts of Interest}

The authors declare that they have no conflicts of interest.

\section{References}

Bekker ZE 1988 - Physiology and biochemistry of mushrooms. MGU, Moscow.

Bermudes D, Gerlach VL, Nealson KH. 1990 - Effects of culture conditions on mycelial growth and luminescence in Panellus stipticus. Mycologia 82, 295-305.

Bondar VS, Puzyr AP, Purtov KV, Medvedeva SE, Rodicheva EK, Gitelson JI. 2011 - The luminescent system of the luminous fungus Neonothopanus nambi. Doklady Biochemistry and Biophysics 438, 138-140.

Bondar V, Shimomura O, Gitelson J. 2012 - Luminescence of higher mushrooms (review). Journal of Siberian Federal University. Biology5 (Biology 4), 331-351.

Bondar VS, Rodicheva E, Medvedeva SE, Tyulkova NA, Tyaglik AB, Shpak BA, Gitelson JI. 2013 - On luminescence mechanism of Neonothopanus nambi mushroom. Doklady Biochemistryand Biophysics 449(2), 223-227.

Dao TV 2009 - Pilot culturing of a luminous mushroom Omphalotus af. illudent (Neonothopanus nambi). Biotechnology 6, 29-37.

Desjardin DE, Oliveira AG, Stevani CV. 2008 - Fungi bioluminescence revisited. Photochemical \& Photobiological Sciences 7, 170-182.

Desjardin DE, Perry BA, Lodge DJ, Stevani CV, Nagasawa E. 2010 - Luminescent Mycena: new and noteworthy species. Mycologia 102(2), 459-477.

Endo M, Kajiwara M, Nakanishi K. 1970 - Fluorescent constituents and cultivation of Lampteromyces japonicus. Journal of the Chemical Society D: Chemical Communication 309-310.

Harvey EN. 1952 - Bioluminescence. Academic Press. New York. 649 p.

Harvey EN. 1957 - A history of luminescence from the earliest times until 1900. J.H. Furst Company, Baltimore, Maryland. 768 p.

Kamzolkina OV, Danilov VS, Egorov NS. 1983 - Nature of luciferase from the bioluminescent fungus Armillariella mellea. Doklady: biochemistry-Akademiia nauk SSSR (USA). 271, 750-752.

Mane VJ, Patil SS, Syed AA, Baig MM. 2007 - Bioconversion of low quality lignocellulosic agricultural wastes into edible protein Pleurotus sajor-caju (Fr.) Singer Journal of Zhejiang University of Science B 8(10), 745-751.

Mendes LF, Bastos EL, Desjardin DE, Stevani CV. 2008 - Influence of culture conditions on mycelial growth and bioluminescence of Gerronema viridilucens. FEMS Microbiology Letters 282, 132-139.

Mihail JD, Bruhn JN. 2007 - Dynamics of bioluminescence by Armillaria gallica, A. mellea and A. tabescens. Mycologia 99(3), 341-350. 
Niitsu H, Hanyuda N, Sugiyama Y. 2000 - Cultural properties of a luminous mushroom, Mycena chlorophos. Mycoscience 41(6), 551-558.

O'Kane DJ, Lingle WL, Porter D, Wampler JE. 1990 - Spectral analysis of the bioluminescence of Panellus stipticus. Mycologia 82, 607-616.

Prasher IB, Chandel VC, Ahluwalia AS. 2012 - Influence of culture conditions on mycelial growth and luminescence of Panellus stipticus (bull.) P. Karst. Journal of Research in Biology-JRB 2(3), 152-159.

Psurtseva NV, Kiyashko AA, Gachkova EJ, Belova NV. 2007 - Basidiomycetes Culture Collection Le (Bin). Catalogue of Strains. 2-th issue. KMK, St. Petersburg.

Shimomura O 2006 - Bioluminescence: chemical principles and methods. World Scientific Publishing Co. Pte. Ltd. pp 266-300.

Vydryakova GA, Psurtseva NV, Belova NV, Pashenova NV, Gitelson JI. 2009 - Luminous mushrooms and prospects of their use. Mikologiya i Fitopatologiya 43, 369-376.

Weitz HJ, Ballard AL, Campbell CD, Killham K. 2001 - The effect of culture conditions on the mycelial growth and luminescence of naturally bioluminescent fungi. FEMS Microbiology Letters 202, 165-170.

Weitz HJ, Colin D, Campbell CD, Killham K. 2002 - Development of a novel, bioluminescence based, fungal bioassay for toxicity testing. Environmental Microbiology 4, 422-429. 